\section{Kidney \\ Blood Pressure \\ Research}

Kidney Blood Press Res 2014;39:658-667

DOI: $10.1159 / 000368476$

Published onine: December 19, 2014

(C) 2014 S. Karger AG, Basel

www.karger.com/kbr

Accepted: December 05, 2014

1423-0143/14/0396-0658\$39.50/0

This is an Open Access article licensed under the terms of the Creative Commons AttributionNonCommercial 3.0 Unported license (CC BY-NC) (www.karger.com/OA-license), applicable to the online version of the article only. Distribution permitted for non-commercial purposes only.

\title{
Aortic Arch Calcification Predicts Cardiovascular and All-Cause Mortality in Maintenance Hemodialysis Patients
}

\author{
Mizuki Komatsu ${ }^{a} \quad$ Masayuki Okazakia Ken Tsuchiya ${ }^{b} \quad$ Hiroshi Kawaguchia \\ Kosaku Nittab
}

aDepartment of Nephrology, Jyoban Hospital, Iwaki-city, Fukushima; 'bepartment of Medicine, Kidney Center, Tokyo Women's Medical University, Shinjuku-ku, Tokyo, Japan

\section{Key Words}

Chest radiography $\bullet$ Aortic calcification $\bullet$ Hemodialysis $\bullet$ Mortality $\bullet$ Cardiovascular disease

\begin{abstract}
Background/Aim: Vascular calcification is associated with cardiovascular risk in maintenance hemodialysis (MHD) patients. Previous reports have shown that simple assessment of aortic arch calcification (AoAC) using plain radiography is associated with cardiovascular mortality in the general population. We conducted a prospective study to investigate factors associated with the presence at baseline and progression of AoAC in MHD patients and examined its prognostic value in a short-term outcome. Methods: We prospectively evaluated chest X-rays in 301 asymptomatic MHD patients. The extent of AoAC was divided into three Grades $(0,1$, $2+3$ ). Demographic data including age, gender, dialysis vintage, co-morbidity and biochemical data were assessed and the patients were then followed for 3 years. Results: AoAC was observed in 126 patients (41.9\%) as Grade 0, in 112 patients (37.2\%) as Grade 1, and in 63 patients (20.9\%) as Grade 2 and 3 at baseline. An increase in the severity of calcification was associated with older male patients who had lower serum albumin levels. During the followup period of 3 years, multivariate Cox proportional hazards analysis revealed that high-grade calcification was associated with cardiovascular and all-cause mortality. Patients with AoAC were associated with a worse outcome in survival analysis and the grade of AAC also influenced their survival. Moreover, all-cause death rates were significantly higher in the progression groups than in the non-progression groups. Conclusions: The presence and progression of AoAC assessed by chest X-ray were independently associated with mortality in MHD patients. Regular follow-up by chest X-ray could be a simple and useful method to stratify mortality risk in MHD patients.
\end{abstract}




\section{Kidney Blood Pressure Research}

Kidney Blood Press Res 2014;39:658-667

DOI: 10.1159/000368476
Publisned onIIne: vecember 19, 2014

(C) 2014 S. Karger AG, Basel

www.karger.com/kbr

Komatsu et al.: Aortic Arch Calcification and Mortality in Hemodialysis

\section{Introduction}

Cardiovascular disease is the major cause of death in patients with end-stage renal disease (ESRD) in Japan [1]. Since traditional risk factors, such as advanced age, hypertension, diabetes, smoking, and dyslipidemia, cannot fully account for the high prevalence of cardiovascular disease, uremia-related factors have been implicated in the pathogenesis of cardiovascular disease in ESRD patients [2]. Vascular calcification in the coronary arteries and the aorta has been recognized as an important risk factor for cardiovascular disease in maintenance hemodialysis (MHD) patients [3]. Recently, accumulating evidence has shown that disturbances in calcium-phosphate metabolism play a pivotal role in cardiovascular disease, partly via the development of vascular calcification [4, 5]

A previous study has demonstrated the association between the presence and extent of vascular calcification and outcome in the dialysis population [5]. The extent of vascular calcification can be quantified with electron beam computer tomography (EBCT) [6] and multi-slice computer tomography (MSCT) [7]. Even though EBCT and MSCT are well-validated noninvasive imaging methods that are considered the golden standard for quantifying vascular calcification, these methods cannot be routinely performed due to the relatively high cost of testing and exposure to a high radiation dose. Plain radiography is a convenient and inexpensive tool for the identification of vascular calcification. In the general population, aortic arch calcification (AoAC) identified in chest radiography has been shown to correlate with cardiovascular mortality [8].

We have recently reported the validity and usefulness of assessment of AoAC grade, as determined by a simple chest X-ray $[9,10]$. AoAC grade was significantly associated with clustering of traditional risk factors. However, it is still unknown whether AoAC grade is a sensitive predictor of cardiovascular and all-cause mortality. The purpose of the present study was to determine the presence and progression of AoAC grade as an independent predictor of cardiovascular and all-cause mortality in MHD patients.

\section{Materials and Methods}

\section{Study population}

There were 356 MHD patients (dialysis duration > 6 months) at Jyoban Hospital Kidney Center, Fukushima, Japan, on January 2011. Of these, patients with acute illness, significant infection, or malignancy were excluded. Of the remaining patients, 301 patients (male/female $=198 / 103$, mean age $63.8 \pm 12.2$ years) gave informed consent for evaluating a chest radiograph, as described next, and were investigated in the present study. The underlying causes of ESRD were diabetes $(n=126)$, chronic glomerulonephritis $(n=$ $95)$, hypertension $(n=70)$, polycystic kidney $(n=6)$, and unknown etiology $(n=4)$. The enrolled patients underwent stable regular hemodialysis using bicarbonate dialysate.

At baseline, a chest radiograph of each patient was obtained. They comprised a non-selected sample of patients from the Kidney Center in Jyoban Hospital without a selection bias. All patients completed a detailed health history questionnaire just prior to follow-up of cardiovascular and all-cause mortality. However, we could not rule out the asymptomatic patients with coronary disease because of no history of coronary angiography. Patients then were followed up for 3 years, and relationships between baseline data and outcomes were assessed statistically. Data and causes of death were obtained by reviewing hospital record forms. In patients who moved to other dialysis units, we reviewed questionnaire forms filled out by the attending physicians at the Kidney Center. This study was in compliance with the Declaration of Helsinki and was approved by the ethics review committee of the Jyoban Hospital.

\section{Assessment of aortic arch calcification}

We performed a retrospective review of 301 patients undergoing dialysis therapy. Two radiologists (one specializing in chest radiography) independently reviewed all chest radiographs obtained from MHD patients studied. Radiographs were assessed for the presence of AoAC using a specific scale as previously described [9]. The scale which was divided into 16 circumferences was attached to the aortic arch on chest 


\section{Kidney \\ Blood Pressure Research}

radiography and then the number of sectors with calcification was divided by 16 . Aortic arch calcification score (AoACS) was calculated after multiplication by 100 to express the results as a percentage. This value was used as the indicator of the AoAC. Our previous study confirmed that AoACS was highly correlated with AoAC volume evaluated by MSCT $(r=0.635, p<0.001)$ [9].

AoAC extent was divided into four grades according to the following categorization. Briefly, we scored the extent of calcification in the aortic arch as follows: grade 0 , AoACS $=0 \%$; grade 0 , AoACS $=1-24 \%$; grade 1, AoACS $=25-49 \%$; grade 3, AoACS $>50 \%$. Progression of AoAC was defined as an increase in AoACS on the follow-up chest X-ray at 1 year, 2 years, and 3 years after enrollment.

\section{Laboratory and hemodynamic measurements}

Blood was drawn just before starting a dialysis session in a fasting state. Serum albumin, calcium, phosphate, total cholesterol, high-density lipoprotein (HDL) cholesterol, triglyceride, blood sugar, and C-reactive protein (CRP) and the concentration of hemoglobin were measured by using routine laboratory methods. Mean values of 3 measurements during the 3 months before chest radiography were used for analysis. Serum intact parathyroid hormone (PTH) was measured once at the time of radiography. Serum calcium levels were adjusted using the formula [calcium + (4-albumin)].

Clinical status of all subjects was evaluated by means of routine clinical examination before the regular HD session. Systolic and diastolic blood pressures were measured with a mercury sphygmomanometer with the patient in the supine position after 10 to 15 minutes of rest, and mean values for 1 month were used for the analysis. Hypertension was defined as predialysis systolic blood pressure greater than $140 \mathrm{mmHg}$, diastolic blood pressure greater than $90 \mathrm{mmHg}$, or if they were using any antihypertensive therapy.

\section{Clinical outcomes}

Subjects in this study were continuously monitored for the occurrence of cardiovascular events and death. In the present study, the cardiovascular events according to our definition were specified clearly as angina pectoris (criteria of angina pectoris were defined by presence of chest symptom and/or typical ST-T change in ECG), myocardial infarction, cerebrovascular disease (transient ischemic attack, stroke, cerebral hemorrhage), heart failure, and cardiovascular death. Individual diagnoses were classified according to the 9th International Classification of Disease (ICD-9) codes.

\section{Statistical analysis}

Continuous variables were expressed as means \pm SD and categorical variables as percentages. Differences between the groups were analyzed using ANOVA for continuous variables and $\chi^{2}$ test for categorical variables. Variables relevant to survival were identified by the univariate Cox proportional hazards method. Significant variables were then selected for further analysis using multivariate Cox proportional hazard models. The Kaplan-Meier method was used to estimate survival probabilities using the log-rank test. The impact of AoAC progression on patient outcome was also examined by the KaplanMeier analysis. All analyses were performed using JMP for Windows (version 11). A p value $<0.05$ was considered statistically significant.

\section{Results}

\section{Baseline Characteristics of the Study Population}

The mean age of the study population was $63.8 \pm 12.2$ years. The average dialysis vintage was $5.8 \pm 6.4$ years. Male patients accounted for $66 \%$. About $40 \%$ of the patients had diabetes. A total of 301 patients were categorized 126 as Grade 0 (41.9\%), 112 as Grade 1 (37.2\%), and 63 as Grade 2 and 3 (20.9\%). Table 1 shows the baseline clinical characteristics of all patients according to the severity of AoAC. There was no significant difference in dialysis vintage, and presence of diabetes and hypertension among all groups. The patients with severe calcification were older than those without calcification $(P<0.0001)$, and a significant increase in male gender $(\mathrm{P}<0.001)$. We did not find significant differences in dialysis adequacy as assessed by Kt/V. Patients with AoAC had lower serum albumin levels $(P=0.0368)$. No significant differences in serum levels in phosphorus, calcium and intact 


\section{Kidney Blood Pressure Research}

Kidney Blood Press Res 2014;39:658-667

DOI: $10.1159 / 000368476$

Publisned onIIne: vecember 19, 2014

(C) 2014 S. Karger AG, Base

www.karger.com/kbr

Komatsu et al.: Aortic Arch Calcification and Mortality in Hemodialysis

Table 1. Baseline demographic and biochemical data of the hemodialysis patients

\begin{tabular}{lccccc}
\hline & Grade 0 & Grade 1 & Grade 2+3 & ALL & P-value \\
\hline Patient, n (\%) & $126(41.9 \%)$ & $112(37.2 \%)$ & $63(20.9 \%)$ & $301(100 \%)$ & \\
Dialysis vintage, years & $5.0 \pm 6.4$ & $6.3 \pm 6.9$ & $6.3 \pm 5.2$ & $5.8 \pm 6.4$ & 0.2261 \\
Age, years & $58.3 \pm 13.7$ & $66.3 \pm 9.2$ & $70.2 \pm 8.6$ & $63.8 \pm 12.2$ & $<0.0001$ \\
Gender, M/F & $98 / 28$ & $63 / 49$ & $37 / 26$ & $198 / 103$ & 0.0009 \\
Diabetes, n (\%) & $53(42.1 \%)$ & $58(51.8 \%)$ & $34(54.0 \%)$ & $145(48.2 \%)$ & 0.1905 \\
Hypertension, n (\%) & $106(84.1 \%)$ & $91(81.3 \%)$ & $54(85.7 \%)$ & $251(83.4 \%)$ & 0.7170 \\
KT/V & $1.4 \pm 0.2$ & $1.4 \pm 0.3$ & $1.4 \pm 0.2$ & $1.4 \pm 0.2$ & 0.2526 \\
Hemoglobin, g/dl & $10.8 \pm 1.2$ & $10.8 \pm 1.1$ & $10.5 \pm 1.1$ & $10.7 \pm 1.1$ & 0.3024 \\
C-reactive protein, mg/dl & $0.3 \pm 0.6$ & $0.4 \pm 0.8$ & $0.6 \pm 1.0$ & $0.4 \pm 0.8$ & 0.0917 \\
Albumin, g/dl & $3.7 \pm 0.3$ & $3.7 \pm 0.3$ & $3.6 \pm 0.3$ & $3.7 \pm 0.3$ & 0.0368 \\
Corrected calcium, mg/dl & $9.1 \pm 0.7$ & $9.1 \pm 0.6$ & $9.2 \pm 0.5$ & $9.1 \pm 0.6$ & 0.3077 \\
Phosphorus, mg/dl & $5.1 \pm 1.6$ & $4.9 \pm 1.6$ & $5.0 \pm 1.7$ & $5.0 \pm 1.6$ & 0.6378 \\
Intact PTH, pg/dl & $139.6 \pm 159.6$ & $121.3 \pm 111.2$ & $122.9 \pm 102.3$ & $129.3 \pm 132.0$ & 0.5188 \\
Total cholesterol, mg/dl & $145.2 \pm 28.6$ & $153.9 \pm 33.2$ & $153.3 \pm 30.2$ & $150.1 \pm 30.9$ & 0.0598 \\
HDL cholesterol, mg/dl & $44.0 \pm 14.0$ & $48.3 \pm 15.5$ & $45.3 \pm 13.3$ & $45.9 \pm 14.5$ & 0.0760 \\
Triglyceride, mg/dl & $119.0 \pm 74.6$ & $114.3 \pm 79.3$ & $107.3 \pm 76.7$ & $114.8 \pm 76.7$ & 0.6122 \\
Non-HDL cholesterol, mg/dl & $101.1 \pm 29.1$ & $105.7 \pm 32.4$ & $108.0 \pm 30.6$ & $104.3 \pm 30.7$ & 0.2901 \\
RAS inhibitors & $66(52.4 \%)$ & $52(46.4 \%)$ & $34(54.0 \%)$ & $152(50.5 \%)$ & 0.5422 \\
CaCO3 & $88(69.8 \%)$ & $83(74.1 \%)$ & $46(73.0 \%)$ & $217(72.1 \%)$ & 0.7520 \\
Non-Ca phosphate binders & $65(51.6 \%)$ & $61(54.5 \%)$ & $36(57.1 \%)$ & $162(53.8 \%)$ & 0.7591 \\
Active Vitamin D3 & $70(55.6 \%)$ & $56(50.0 \%)$ & $38(60.3 \%)$ & $164(54.5 \%)$ & 0.4003 \\
\hline Values are expressed as means \pm SD, unless otherwise specified. PTH = parathyroid hormone; HDL = high-density \\
lipoprotein; RAS = renin-angiotensin system; Non-Ca phosphate binder; non-calcium containing phosphate binder. \\
\hline
\end{tabular}

PTH were shown and the lipid profile did not differ. There was no significant difference in the prescription of renin-angiotensin system (RAS) inhibitor, $\mathrm{CaCO3}$, non-calcium containing phosphate binders and active vitamin D3.

\section{Risk Factors Associated with Mortality}

During a follow-up period of 3 years, there were $65(21.6 \%)$ deaths in the study population. Cardiovascular death was detected in 43 deaths, which accounted for $66.2 \%$ of all deaths, followed by infectious disease $(23.1 \%)$, malignancy $(6.2 \%)$ and gastrointestinal bleeding (4.6\%). As shown in Table 2, univariate analysis demonstrated that age, diabetes, hypertension, prescription of active vitamin D3 and AoAC (Grade 2 and 3) were significantly associated with cardiovascular mortality. In multivariate analysis, diabetes, hypertension, prescription of active vitamin D3 and Grade 2and 3 AoAC were independently associated with cardiovascular mortality. Table 3 shows factors associated with all-cause mortality. Age, diabetes, serum levels of albumin and non-HDL cholesterol, prescription of active vitamin D3 and AoAC (Grade 2 and 3) were significant factors associated with all-cause mortality. Multivariate analysis demonstrated that age, diabetes, serum levels of albumin and non-HDL cholesterol and Grade 2 and 3 AoAC were independent factors related to overall survival.

\section{Survival Analysis}

Kaplan-Meier survival analysis of cardiovascular mortality is shown in Fig. 1. Patients without AoAC had less cardiovascular survival than those with AoAC $(P=0.0406)$. A higher mortality rate was observed in patients with high-grade AoAC (Grade 2+3). Fig. 2 displays the survival curve of different groups according to the severity of AoAC. Significant 


\section{Kidney Blood Pressure Research}

Table 2. Univariate and multivariate Cox proportional hazards analysis for cardiovascular mortality

\begin{tabular}{|c|c|c|c|c|c|c|}
\hline & & Univariate & & & Multivariate & \\
\hline & HR & $95 \% \mathrm{CI}$ & P-value & HR & $95 \% \mathrm{CI}$ & $\mathrm{P}$-value \\
\hline Dialysis vintage & 1.007 & $0.939-1.064$ & 0.8228 & & & \\
\hline Age & 1.038 & $1.000-1.081$ & 0.0488 & 1.035 & 0.993-1.082 & 0.1061 \\
\hline Male gender & 1.502 & $0.624-4.163$ & 0.3772 & & & \\
\hline Diabetes & 4.151 & $1.656-12.572$ & 0.0018 & 4.551 & $1.776-14.002$ & 0.0011 \\
\hline Hypertension & 0.354 & $0.154-0.880$ & 0.0269 & 0.322 & $0.137-0.815$ & 0.0185 \\
\hline $\mathrm{KT} / \mathrm{V}$ & 0.376 & $0.074-2.069$ & 0.2611 & & & \\
\hline Hemoglobin & 1.039 & $0.728-1.508$ & 0.8386 & & & \\
\hline C-reactive protein & 1.282 & $0.831-1.712$ & 0.2199 & & & \\
\hline Albumin & 0.336 & $0.113-1.117$ & 0.0741 & & & \\
\hline Calcium & 1.196 & $0.607-2.264$ & 0.5966 & & & \\
\hline Phosphorus & 0.919 & $0.708-1.184$ & 0.5189 & & & \\
\hline Intact PTH & 0.997 & $0.992-1.001$ & 0.2307 & & & \\
\hline Total cholesterol & 1.196 & $0.607-2.264$ & 0.7011 & & & \\
\hline HDL cholesterol & 1.018 & $0.991-1.043$ & 0.1903 & & & \\
\hline Triglyceride & 1.000 & 0.993-1.005 & 0.9228 & & & \\
\hline Non-HDL cholesterol & 0.993 & $0.979-1.006$ & 0.3046 & & & \\
\hline RAS inhibitor & 0.620 & $0.258-1.414$ & 0.2578 & & & \\
\hline $\mathrm{CaCO} 3$ & 1.111 & $0.461-3.079$ & 0.8234 & & & \\
\hline Non-Ca Phosphate binder & 0.919 & $0.403-2.118$ & 0.8405 & & & \\
\hline Active Vitamin D3 & 0.287 & $0.103-0.690$ & 0.0047 & 0.305 & $0.108-0.748$ & 0.0087 \\
\hline AoAC & & & & & & \\
\hline Grade 1 & 2.838 & $1.053-8.920$ & 0.0390 & 1.731 & $0.616-5.623$ & 0.3065 \\
\hline Grade $2+3$ & 4.636 & $2.794-9.149$ & 0.0011 & 2.629 & $1.455-5.124$ & 0.016 \\
\hline
\end{tabular}

differences were detected between patients with and without AoAC $(\mathrm{P}=0.0373)$. There was no significant difference between Grade 1 and Grade 2+ 3 .

Progression of AoAC as an Independent Risk Factor for Mortality

Kaplan-Meier analysis was used to determine the prognostic value of AoAC progression on mortality. MHD patients with AoAC progression had not significantly differences in cardiovascular mortality $(\mathrm{P}=0.1830$, Fig. 3 ). However, AoAC progression was significantly associated with higher all-cause mortality ( $\mathrm{P}=0.0251$, Fig. 4).

\section{Discussion}

The results of the present study demonstrated a high prevalence of vascular calcification in MHD patients: more than 50\% of the patients presented with AoAC and about one fourth of them had high-grade calcification. AoAC was associated with old age and male gender. Serum albumin levels were significant associates of AoAC. Furthermore, we found that AoAC progression was significantly associated with higher all-cause mortality but not cardiovascular mortality.

Vascular calcification has been considered a risk factor of cardiovascular mortality in ESRD patients. Arterial calcification increase stiffness and reduce elasticity of large arteries 


\section{Kidney Blood Pressure Research}

Table 3. Univariate and multivariate Cox proportional hazards analysis for all-cause mortality

\begin{tabular}{|c|c|c|c|c|c|c|}
\hline & \multicolumn{3}{|c|}{ Univariate } & \multicolumn{3}{|c|}{ Multivariate } \\
\hline & HR & $95 \% \mathrm{CI}$ & $\mathrm{P}$-value & HR & $95 \% \mathrm{CI}$ & P-value \\
\hline Dialysis vintage & 1.013 & $0.963-1.056$ & 0.6038 & & & \\
\hline Age & 1.058 & $1.026-1.096$ & 0.0002 & 1.060 & $1.022-1.102$ & 0.0013 \\
\hline Male gender & 1.485 & $0.746-3.215$ & 0.2690 & & & \\
\hline Diabetes & 2.835 & $1.445-5.963$ & 0.0021 & 2.615 & $1.306-5.609$ & 0.0061 \\
\hline Hypertension & 0.608 & $0.299-1.362$ & 0.2125 & & & \\
\hline $\mathrm{KT} / \mathrm{V}$ & 0.393 & $0.109-1.482$ & 0.1678 & & & \\
\hline Hemoglobin & 0.918 & 0.703-1.219 & 0.5490 & & & \\
\hline C-reactive protein & 1.312 & $0.968-1.643$ & 0.0751 & & & \\
\hline Albumin & 0.202 & $0.091-0.481$ & 0.0004 & 0.327 & $0.134-0.859$ & 0.0242 \\
\hline Calcium & 1.400 & $0.839-2.280$ & 0.1939 & & & \\
\hline Phosphorus & 0.972 & $0.795-1.181$ & 0.7784 & & & \\
\hline Intact PTH & 0.998 & 0.993-1.001 & 0.1481 & & & \\
\hline Total cholesterol & 0.994 & $0.983-1.004$ & 0.2393 & & & \\
\hline HDL cholesterol & 1.019 & 0.999-1.039 & 0.0678 & & & \\
\hline Triglyceride & 0.997 & 0.991-1.002 & 0.2207 & & & \\
\hline Non-HDL cholesterol & 0.989 & 0.977-0.999 & 0.0363 & 0.984 & $0.972-0.997$ & 0.0111 \\
\hline RAS inhibitor & 0.868 & $0.455-1.643$ & 0.6618 & & & \\
\hline $\mathrm{CaCO} 3$ & 0.962 & $0.490-2.023$ & 0.9137 & & & \\
\hline Non-Ca Phosphate binder & 0.684 & $0.356-1.295$ & 0.2433 & & & \\
\hline Active Vitamin D3 & 0.420 & 0.209-0.807 & 0.0089 & 0.398 & $0.196-0.772$ & 0.0062 \\
\hline \multicolumn{7}{|l|}{ AoAC } \\
\hline Grade 1 & 2.006 & $0.934-4.545$ & 0.0747 & 1.230 & $0.559-2.850$ & 0.6112 \\
\hline Grade $2+3$ & 3.409 & $2.015-5.781$ & 0.0261 & 1.699 & $1.052-2.680$ & 0.0222 \\
\hline
\end{tabular}

$\mathrm{PTH}=$ parathyroid hormone; $\mathrm{HDL}=$ high-density lipoprotein; $\mathrm{RAS}=\mathrm{RAS}=$ renin angiotensin system; AoAC $=$ aortic arch calcification .

such as the aorta, which may result in substantial mortality and morbidity by impairing cardiovascular hemodynamics and vascular compliance [11]. Several imaging methods such as EBCT and MSCT are available to detect cardiovascular calcification in HD patients. These methods differ in the sensitivity, availability and cost $[12,13]$. The KDIGO clinical practice guideline recommends plain X-ray films of the lumbar spine for the detection and assessment of cardiovascular calcification in HD patients [14]. The AoAC identified in plain chest $\mathrm{X}$-ray is associated with an increased risk of coronary artery disease and is linked to cardiovascular risk factors such as age, hypertension, dyslipidemia and diabetes mellitus in the
Fig. 1. Kaplan-Meier analysis for cardiovascular mortality of 301 maintenance hemodialysis patients.

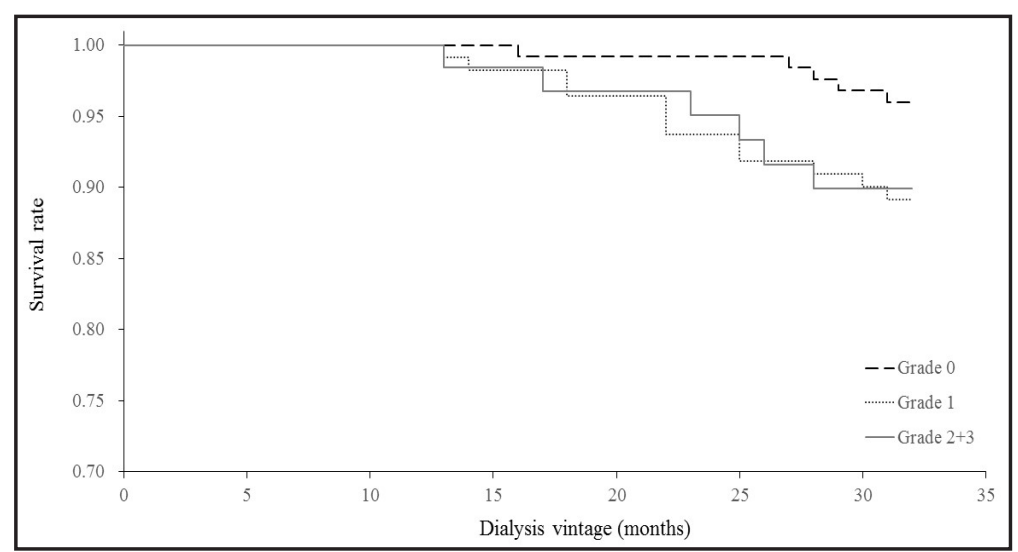




\section{Kidney \\ Blood Pressure Research}

general population $[15$, 16]. Moreover, compared with traditional risk factors, AoAC is an independent determinant of cardiovascular outcome [17]. The present study supports the additional value of plain chest X-ray films in MHD patients to predict their short-term outcome.

If the level of calcification in the aorta predicts future cardiovascular events, which area in the abdominal aorta or aortic arch is favorable and sensitive to evaluate the extent of calcification should be considered. First, there is much strong evidence that radiographic abdominal aortic calcification predicts cardiovascular events in huge populations during longterm follow-up. Okuno et al. also reported that the presence of abdominal aortic calcification is significantly associated with cardiovascular mortality in 515 HD patients (hazard ratio, 2.07; 95\% confidence interval, 1.21-3.56; $P<$ 0.01) [18], suggesting valuable evaluation of abdominal aortic calcification using abdominal X-ray. On the other hand, it is unclear whether the calcified level in the aortic arch is also a good predictor similar to the extent of calcification in the abdominal aorta. There are relatively few reports regarding the predictive value of AoAC against cardiovascular events, although many previous studies have shown a positive association of cardiovascular events with abdominal aortic calcification. One possible explanation is that it is not easier to evaluate calcium deposition semi-quantitatively in the aortic arch as compared to that in the abdominal aorta.
Fig. 2. Kaplan-Meier analysis for all-cause mortality of 301 maintenance hemodialysis patients.

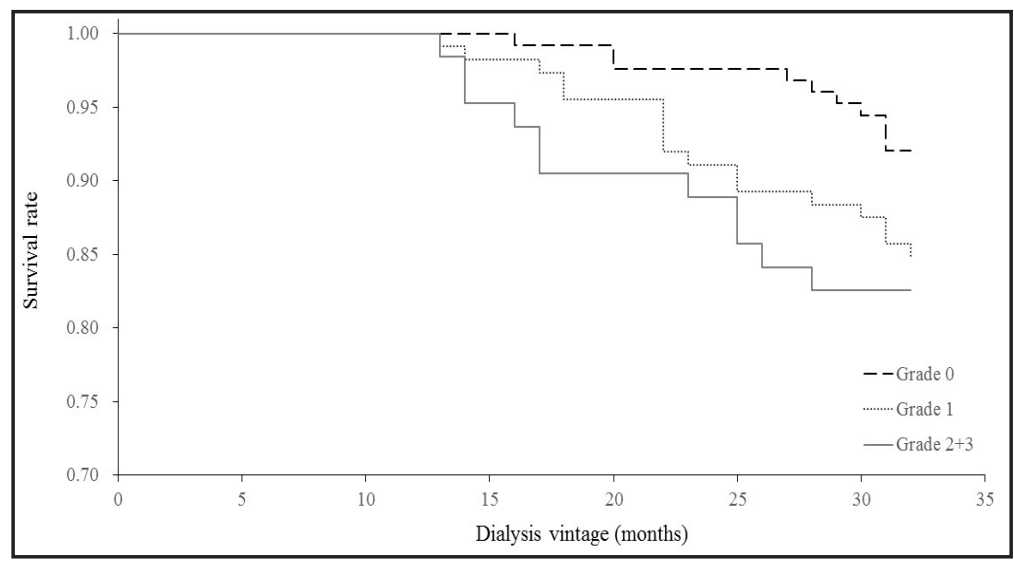

Fig. 3. Kaplan-Meier analysis of aortic arch calcification progression for cardiovascular mortality of 301 maintenance hemodialysis patients. Prog = progression.

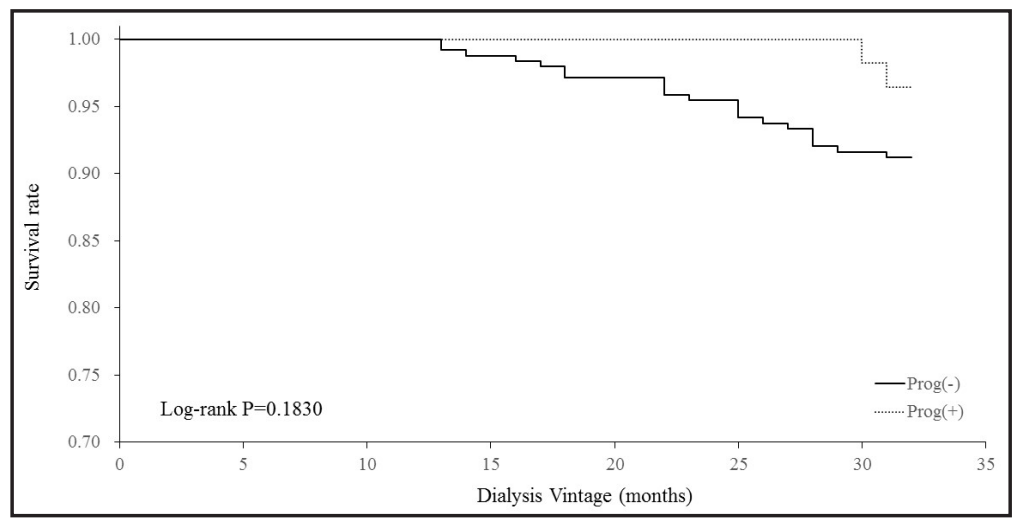

Fig. 4. Kaplan-Meier analysis of aortic arch calcification progression for allcause mortality of 301 hemodialysis patients. Prog = progression.

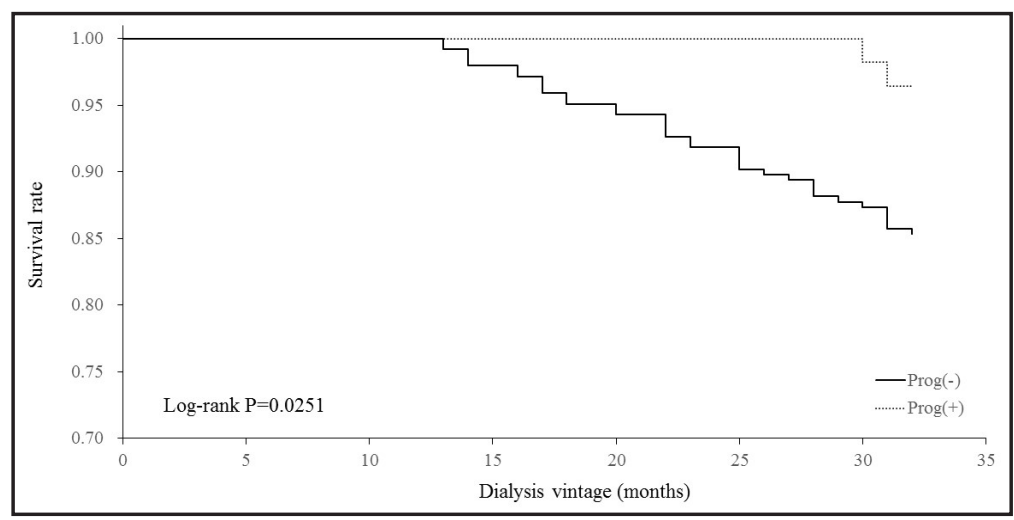




\section{Kidney Blood Pressure Research}

\begin{tabular}{l|l}
\hline Kidney Blood Press Res 2014;39:658-667 \\
\hline DOI: $10.1159 / 000368476$ & (c) 2014 S. Karger AG, Basel
\end{tabular}

Published online: December 19, 2014

www.karger.com/kbr

Komatsu et al.: Aortic Arch Calcification and Mortality in Hemodialysis

A report handling a population-based cohort has showed that the presence of AoAC was independently associated with an increased risk of cardiovascular mortality (hazard ratio, 2.556; 95\% confidence interval, 1.006-6.490; $P<0.05$ ) [19], suggesting that the present results are consistent with this evidence. However, that study evaluated whether AoAC was present or absent using chest X-ray without considering the extent of calcification. There are several advantages and differences of our study as compared to the previous report. Our assessment by AoAC grading certified a strong predictive value of higher AoAC grade (grades $2+3$ ) against incident cardiovascular events more precisely. However, AoAC grade 1 had no statistical significance of predictive value compared to grade 0 . This result suggests that trivial calcium deposition in the aortic arch only may not be a noticeable cardiovascular risk. Hashimoto et al. recently confirmed that the AoAC grade was significantly correlated with the calcified level in the abdominal aorta using two independent non-invasive examinations [20]. In fact, huge differences in the hemodynamic state are found between patients with trivial AoAC and those with severe AoAC. Therefore, it is recommended to consider the AoAC grade in routine clinical work of dialysis therapy.

The results of the present study have shown the association between AoAC at baseline and short-term outcome, indicating the importance of screening for cardiovascular calcification in MHD patients. In multivariate analysis, patients with high-grade AoAC had a significant association with cardiovascular death and all-cause mortality. Among factors relevant to survival, diabetes was independently associated with both cardiovascular and all-cause mortality. These data are almost identical with those recently reported by Lee et al. [21]. However, Lee et al. did not include the prescription of RAS inhibitors, CaCO3, noncalcium phosphate binders and active vitamin D as confounders. Especially, the prescription of active vitamin D is associated with cardiovascular and all-cause mortality (Table 2 and $3)$. It has been reported that pre-dialysis fasting glucose levels correlate with short-term outcome in HD patients [22]. Kim et al. have recently reported that diabetes accelerates the progression of AoAC in HD patients [23]. They showed that hyperglycemia mostly associated with type 2 diabetes, was the only significant predictor of vascular calcification in ESRD patients. Recent evidence suggests that medial calcification in diabetes in an active, cellmediated process, similar to that observed in ESRD patients [24].

One of the important findings in the present study is the association between prescription of active vitamin D3 and short-term outcome. The use of active vitamin D3 is an independent predictor for cardiovascular and all-cause mortality. We previously demonstrated that alfacalcidol therapy was associated with a significantly lower risk of cardiovascular and all-cause mortality in chronic HD patients [25]. Low doses and more physiological doses of active vitamin D have been found to have a cardioprotective effect [26, 27]. We previously reported finding that low-dose oral vitamin D therapy protect HD patients from developing vascular calcification [28]. In a study of 242 HD patients, Shoji et al. [29] reported that HD patients treated with alfacalcidol were at reduced risk of cardiovascular death according to an adjusted Cox model. Naves-Diaz et al. [30] reported that a mean daily oral vitamin D dose below $0.25 \mu \mathrm{g}$ was able to reduce the mortality rate by $53 \%$ in HD patients whose serum PTH levels were below $150 \mathrm{pg} / \mathrm{ml}$, independently of their serum calcium or phosphorus levels. These findings suggest that low dose active vitamin D3 may improve cardiovascular and allcause mortality of HD patients in association with the modulation of vascular calcification.

There are several limitations in the present study. First, evaluation of AoAC is the semiquantitative method, thus this method using four grades to evaluate AoAC is relatively crude. Therefore, the true calcium deposition in the aortic wall may be underestimated. However, our previous study confirmed that AoACS was highly correlated with aortic arch calcification volume evaluated by MSCT [9]. Second, the largely observational design reduced the quality of the study, and the population size in this study was also small. Therefore, a largely prospective study using examinations for AoAC evaluated by both plain chest X-ray and CT is necessary. 


\section{Kidney \\ Blood Pressure Research}

Komatsu et al.: Aortic Arch Calcification and Mortality in Hemodialysis

\section{Conclusion}

The presence and progression of AoAC assessed by chest X-ray were independently associated with mortality in MHD patients. Regular follow-up by chest X-ray could be a simple and useful method to stratify mortality risk in MHD

\section{Disclosure Statement}

We have no conflict of interest to disclose.

\section{Acknowledgements}

This study was in part supported by a Grant-in-Aid from the Japan Promotion Society for Cardiovascular Diseases.

\section{References}

1 Nakai S, Watanabe Y, Masakane I, Wada A, Shoji T, Hasegawa T, Nakamoto H, Yamagata K, Kazama JJ, Fujii N, Shinoda T, Shigematsu T, Marubayashi S, Morita O, Hashimoto S, Suzuki K, Kimata N, Hanafusa N, Wakai K, Hamano T, Ogata S, Tsuchida K, Taniguchi M, Nishi H, Iseki K, Tsubakihara Y: Overview of regular dialysis treatment in Japan (as of 31 December 2011). Ther Apher Dial 2013;17:567-611.

2 Block GA, Klassen PS, Lazarus JM, Ofsthun N, Lowrie EG, Chertow GM: Mineral metabolism, mortality, and morbidity in maintenance hemodialysis. J Am Soc Nephrol 2004;15:2208-2218.

-3 Cannata-Andia JB, Rodriguez-Garcia M, Carrillo-Lopez N, Naves-Diaz M, Diaz-Lopez B: Vascular calcifications: pathogenesis, management, and impact on clinical outcomes. J Am Soc Nephrol 2006;17:S267-S273.

-4 Shanahan CM, Crouthamel MH, Kapustin A, Giachelli CM: Arterial calcification in chronic kidney disease: key roles for calcium and phosphate. Circ Res 2011;109:697-711.

-5 Noordzij M, Cranenburg EM, Engelsman LF, Hermans MM, Boeschoten EW, Brandenburg VM, Bos WJ, Kooman JP, Dekker FW, Ketteler M, Schurgers LJ, Krediet RT, Korevaar JC; NECOSAD Study Group: Progression of aortic calcification is associated with disorders of mineral metabolism and mortality in chronic dialysis patients. Nephrol Dial Transplant 2011;26:1662-1669.

-6 Agatston AS, Janowitz WR, Hildner FJ, Zusmer NR, Viamonte M Jr, Detrano R: Quantification of coronary artery calcium using ultrafast computed tomography. J Am Coll Cardiol 1990;15:827-832.

7 Moe SM, O’Neil KD, Fineberg N, Persohn S, Ahmed S, Garret P, Meyer CA: Assessment of vascular calcification in ESRD patients using spiral CT. Nephrol Dial Transplant 2003;18:1152-1158.

8 Iribarren C, Sidney S, Sternfeld B, Browner WS: Calcification of the aortic arch: risk factors and association with coronary heart disease, stroke, and peripheral vascular disease. JAMA 2000;283:2810-2815.

-9 Ogawa T, Ishida H, Matsuda N, Fujiu A, Matsuda A, Ito K, Ando Y, Nitta K: Simple evaluation of aortic arch calcification by chest radiography in hemodialysis patients. Hemodial Int 2009;13:301-306.

-10 Inoue T, Ogawa T, Ishida H, Ando Y, Nitta K: Aortic arch calcification evaluated on chest X-ray is a strong independent predictor of cardiovascular events in chronic hemodialysis patients. Heart Vessels 2012;27:135-142.

11 London GM, Guerin AP, Marchais SJ, Metivier F, Pannier B, Adda H: Arterial media calcification in end-stage renal disease: impact on all-cause and cardiovascular mortality. Nephrol Dial Transplant 2003;18:17311740 .

12 Bellasi A, Ferramosca E, Muntner P, Ratti C, Wildman RP, Block GA, Raggi P: Correlation of simple imaging tests and coronary artery calcium measured by computed tomography in hemodialysis patients. Kidney Int 2006;70:1623-1628. 


\section{Kidney \\ Blood Pressure Research}

Komatsu et al.: Aortic Arch Calcification and Mortality in Hemodialysis

13 Karohl C, Raggi P: Cardiovascular imaging in patients with chronic kidney disease. Blood Purif 2011;31:130-137.

-14 Kidney Disease: Improving Global Outcomes (KDIGO) CKD-MBD Work Group: KDIGO clinical practice guideline for the diagnosis, evaluation, prevention, and treatment of Chronic Kidney Disease-Mineral and Bone Disorder (CKD-MBD). Kidney Int Suppl 2009:S1-S130.

15 Symeonidis G, Papanas N, Giannakis I, Mavridis G, Lakasas G, Kyriakidis G, Artopoulos I: Gravity of aortic arch calcification as evaluated in adult Greek patients. Int Angiol 2002;21:233-236.

-16 Li J, Galvin HK, Johnson SC, Langston CS, Sclamberg J, Preston CA: Aortic calcification on plain chest radiography increases risk for coronary artery disease. Chest 2002;121:1468-1471.

-17 Iijima K, Hashimoto H, Hashimoto M, Son BK, Ota H, Ogawa S, Eto M, Akishita M, Ouchi Y: Aortic arch calcification detectable on chest X-ray is a strong independent predictor of cardiovascular events beyond traditional risk factors. Atherosclerosis 2010;210:137-144.

- 18 Okuno S, Ishimura E, Kitatani K, Fujino Y, Kohno K, Maeno Y, Maekawa K, Yamakawa T, Imanishi Y, Inaba M, Nishizawa Y: Presence of abdominal aortic calcification is significantly associated with all-cause and cardiovascular mortality in maintenance hemodialysis patients. Am J Kidney Dis 2007;49:417-425.

19 Ogawa T, Ishida H, Akamatsu M, Matsuda N, Fujiu A, Ito K, Ando Y, Nitta K: Progression of aortic arch calcification and all cause-mortality and cardiovascular mortality in chronic hemodialysis patients. Int Urol Nephrol 2010;42:187-194.

20 Hashimoto H, Iijima K, Hashimoto M, Son BK, Ota H, Ogawa S, Eto M, Akishita M, Ouchi Y: Validity and usefulness of aortic arch calcification in chest. J Atheroscler Thomb 2009;16:256-264.

21 Lee CT, Huang CC, Hsu CY, Chiou TT, Ng HY, Wu CH, Kuo WH, Lee YT: Calcification of the aortic arch predicts caldiovascular and all-cause mortality in chronic hemodialysis patients. Cardiorenal Med 2014;4:34-42.

-22 Lee MJ, Shin DH, Kim SJ, Oh HJ, Yoo DE, Ko KI, Koo HM, Kim CH, Doh FM, Park JT, Han SH, Yoo TH, Choi KH, Kang SW: Progression of aortic arch calcification over 1 year is an independent predictor of mortality in incident peritoneal dialysis patients. PLoS One 2012; 7:e48793.

23 Kim HG, Song SW, Kim TY, Kim YO: Risk factors for progression of aortic arch calcification in patients on maintenance hemodialysis and peritoneal dialysis. Hemodial Int 2011;15:460-467.

24 Chen NX, Moe SM: Arterial calcification in diabetes. Curr Diab Rep 2003;3:28-32.

25 Ogawa M, Ogawa T, Inoue T, Otsuka K, Nitta K: Effect of alfacalcidol therapy on the survival of chronic hemodialysis patients. Ther Apher Dial 2012;16:248-253.

$26 \mathrm{Wu}$ J, Garami M, Cheng T, Gardner DG: 1,25(OH)2 vitamin D3, and retinoic acid antagonize endothelinstimulated hypertrophy of neonatal cardiac myocytes. J Clin Invest 1996;97:1577-1588.

27 O'Connell TD, Berry JE, Jarvis AK, Somerman MJ, Simpson RU: 1,25-dihydroxyvitamin D3 regulation of cardiac myocyte proliferation and hypertrophy. Am J Physiol 1997;272:H1751-H1758.

28 Ogawa T, Ishida H, Akamatsu M, Matsuda N, Fujiu A, Ito K, Ando Y, Nitta K: Relation of oral 1alpha-hydroxy vitamin D3 to the progression of aortic arch calcification in hemodialysis patients. Heart Vessels 2010;25:16.

29 Shoji T, Shinohara K, Kimoto E, Emoto M, Tahara H, Koyama H, Inaba M, Fukumoto S, Ishimura E, Miki T, Tabata T, Nishizawa Y: Lower risk for cardiovascular mortality in oral 1alpha-hydroxy vitamin D3 users in haemodialysis population. Nephrol Dial Transplant 2004;19:179-184.

-30 Naves-Diaz M, Alvarez-Hernandez D, Passlick-Deetjen J, Guinsburg A, Marelli C, Rodriguez-Puyol D, Cannata-Andia JB: Oral active vitamin D is associated with improved survival in hemodialysis patients. Kidney Int 2008;74:1070-1078. 
In the article by Komatsu et al., entitled "Aortic Arch Calcification Predicts Cardiovascular and All-Cause Mortality in Maintenance Hemodialysis Patients", published in Kidney and Blood Pressure Research, 2014;39:658-667 (DOI: 10.1159/000368476), is an error on page 664, Figure 3 and Figure 4: Prog (-)Prog(+) are the opposite in the figures.

The correct figures with legends are reproduced here. The authors sincerely apologize for this error.

Fig. 3. Kaplan-Meier analysis of aortic arch calcification progression for cardiovascular mortality of 301 maintenance hemodialysis patients. Prog = progression.

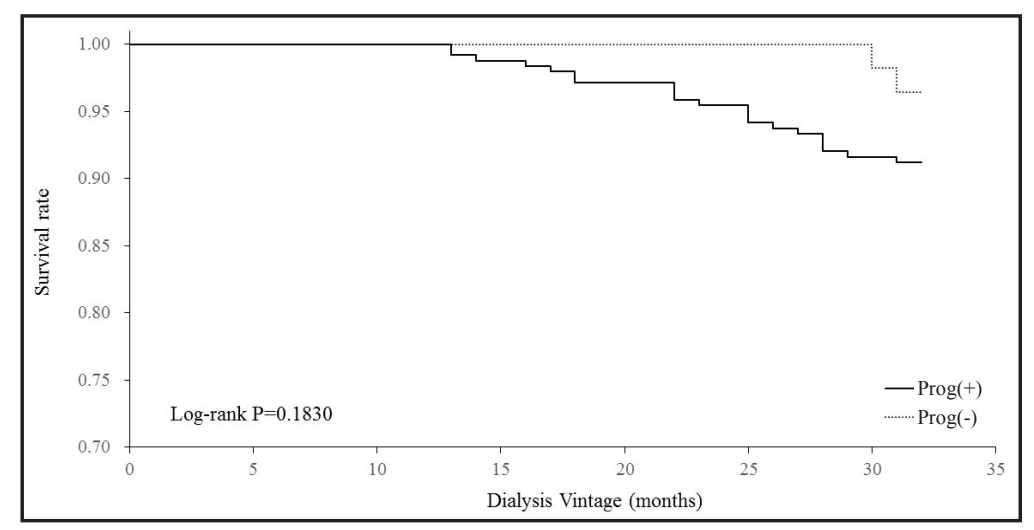

Fig. 4. Kaplan-Meier analysis of aortic arch calcification progression for allcause mortality of 301 hemodialysis patients. Prog = progression.

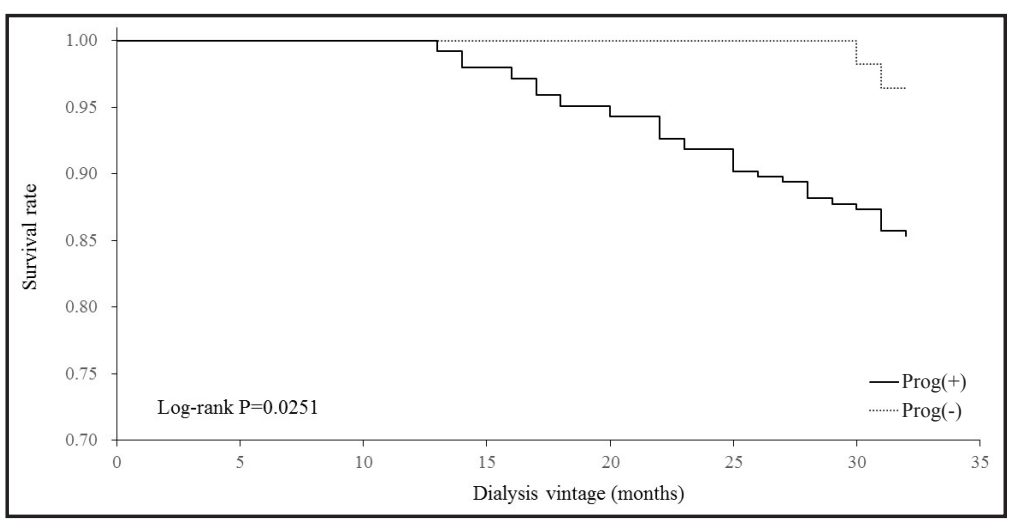

